\title{
On the Origin of Hyperfast Neutron Stars
}

\author{
V.V. Gvaramadze ${ }^{1}$, A. Gualandris ${ }^{2,3}$ \\ and S. Portegies Zwart ${ }^{3}$ \\ ${ }^{1}$ Sternberg Astronomical Institute, Moscow State University, Universitetskij Pr. 13, Moscow \\ 119992, Russia \\ email: vgvaram@mx.iki.rssi.ru \\ ${ }^{2}$ Center for Computational Relativity and Gravitation, Rochester Institute of Technology, 78 \\ Lomb Memorial Drive, Rochester, NY 14623, USA \\ email: alessiag@astro.rit.edu \\ 3 Astronomical Institute 'Anton Pannekoek' and Section Computational Science, Amsterdam \\ University, Kruislaan 403, 1098 SJ, Amsterdam, the Netherlands \\ email: spz@science.uva.nl
}

\begin{abstract}
We propose an explanation for the origin of hyperfast neutron stars (e.g. PSR B1508+55, PSR B2224+65, RX J0822-4300) based on the hypothesis that they could be the remnants of a symmetric supernova explosion of a high-velocity massive star (or its helium core) which attained its peculiar velocity (similar to that of the neutron star) in the course of a strong three- or four-body dynamical encounter in the core of a young massive star cluster. This hypothesis implies that the dense cores of star clusters (located either in the Galactic disk or near the Galactic centre) could also produce the so-called hypervelocity stars - ordinary stars moving with a speed of $\sim 1000 \mathrm{~km} \mathrm{~s}^{-1}$.
\end{abstract}

Keywords. Stars: neutron, pulsars: general, pulsars: individual (B1508+55), galaxies: star clusters, methods: n-body simulations

\section{Introduction}

Recent proper motion and parallax measurements for the pulsar PSR B1508+55 (Chatterjee et al. 2005) gave the first example of a high velocity $\left(1083_{-90}^{+103} \mathrm{~km} \mathrm{~s}^{-1}\right)$ directly measured for a neutron star (NS). A possible way to account for extremely high velocities of NSs $\dagger$ is to assume that they are due to a natal kick or a post-natal acceleration (Chatterjee et al. 2005). In this paper, we propose an alternative explanation for the origin of hyperfast NSs (cf. Gvaramadze 2007) based on the hypothesis that they could be the remnants of symmetric supernova (SN) explosions of hypervelocity stars [HVSs; the ordinary stars moving with extremely high $\left(\sim 1000 \mathrm{~km} \mathrm{~s}^{-1}\right)$ peculiar velocities; e.g. Brown et al. 2005]. A strong argument in support of this hypothesis comes from the fact that the mass of one of the HVSs is $\geqslant 8 M_{\odot}$ (Edelmann et al. 2005) so that this star ends its evolution in a type II SN leading to the production of a hyperfast NS.

\section{Hypervelocity stars and young massive star clusters}

It is believed that the origin of HVSs could be connected to scattering processes involving the supermassive black hole (BH) in the Galactic centre (Hills 1988; Yu \& Tremaine 2003; Gualandris et al. 2005). It is therefore possible that the progenitors of some hyperfast NSs were also ejected from the Galactic centre. The proper motion and parallax

$\dagger$ Other possible examples of hyperfast NSs are PSR B2224+65 (Chatterjee \& Cordes 2004) and RX J0822-4300 (Hui \& Becker 2006). 
measured for PSR B1508+55, however, indicate that this NS was born in the Galactic disk (Chatterjee et al. 2005). The kinematic characteristics of some high-velocity early B stars also suggest that these objects originated in the disk (e.g. Ramspeck et al. 2001). We consider the possibility that the HVSs (including the progenitors of hyperfast NSs) could be ejected not only from the Galactic centre but also from the cores of young $\left(<10^{7}\right.$ yr) massive $\left(\sim 10^{4}-10^{5} M_{\odot}\right)$ star clusters (YMSCs), located either in the Galactic disk or near the Galactic centre (cf. Gualandris \& Portegies Zwart 2007).

\section{Origin of hyperfast neutron stars}

To check the hypothesis that the hyperfast NSs could be the descendants of HVSs which were ejected from the cores of YMSCs, we calculated (see Gvaramadze et al. 2007) the maximum possible ejection speed produced by dynamical processes involving close encounters between: $i$ ) two hard (Heggie 1975) massive binaries (e.g. Leonard 1991), ii) a hard binary and an intermediate-mass $\left(\sim 100-1000 M_{\odot}\right)$ BH (e.g. Portegies Zwart \& McMillan 2002), and iii) a single star and a hard binary intermediate-mass BH (e.g. Gürkan et al. 2006). We find that main-sequence O-type stars cannot be ejected from YMSCs with peculiar velocities high enough to explain the origin of hyperfast NSs, but lower mass main-sequence stars or the stripped helium cores of massive stars could be accelerated to hypervelocities. We find also that the dynamical processes in the cores of YMSCs can produce stars moving with velocities of $\sim 200-400 \mathrm{~km} \mathrm{~s}^{-1}$ which therefore contribute to the origin of high-velocity NSs as well as to the origin of the bound population of halo stars (Ramspeck et al. 2001; Brown et al. 2007).

\section{Acknowledgements}

V.V.G. acknowledges the International Astronomical Union and the Russian Foundation for Basic Research for travel grants. A. G. and S. P. Z. acknowledge support from the Netherlands Organization for Scientific Research (NWO under grant No. 635.000.001 and 643.200.503), the Royal Netherlands Academy of Arts and Sciences (KNAW) and the Netherlands Research School for Astronomy (NOVA).

\section{References}

Brown, W. R., Geller, M. J., Kenyon, S. J., \& Kurtz, M. J. 2005, ApJ 622, L33

Brown, W. R., Geller, M. J., Kenyon, S. J., Kurtz, M. J., \& Bromley, B. C. 2007, ApJ 660, 311

Chatterjee, S., \& Cordes, J. M. 2004, ApJ 600, L51

Chatterjee, S., et al. 2005, ApJ 630, L61

Edelmann, H., Napiwotzki, R., Heber, U., Christlieb, N., \& Reimers, D. 2005, ApJ 634, L181

Gualandris, A., \& Portegies Zwart, S. 2007, MNRAS 376, L29

Gualandris, A., Portegies Zwart, S., \& Sipior, M. S. 2005, MNRAS 363, 223

Gürkan, M. A., Fregeau, J. M., \& Rasio, F. A. 2006, ApJ 640, L39

Gvaramadze, V. V. 2007, A\&A 470, L9

Gvaramadze, V. V., Gualandris, A., \& Portegies Zwart, S. 2007, preprint: astro-ph/0702735

Heggie, D. C. 1975, MNRAS 173, 729

Hills, J. G. 1988, Nat 331, 687

Hui, C. Y., \& Becker, W. 2006, A $\& A$ 457, L33

Leonard, P. J. T. 1991, AJ 101, 562

Portegies Zwart, S. F., \& McMillan, S. L. W. 2002 ApJ 576, 899

Ramspeck, M., Heber, U., \& Moehler, S. 2001, A $\& A$ 378, 907

Yu, Q., \& Tremaine, S. 2003, ApJ 599, 1129 